\title{
Oscillation of higher order nonlinear dynamic equations on time scales
}

\author{
Said R Grace ${ }^{1 *}$, Ravi P Agarwal ${ }^{2}$ and Ağacık Zafer $^{3}$
}

\author{
* Correspondence: srgrace@eng.cu. \\ edu.eg \\ 'Department of Engineering \\ Mathematics, Faculty of \\ Engineering, Cairo University, \\ Oman, Giza 12221, Egypt \\ Full list of author information is \\ available at the end of the article
}

\begin{abstract}
Some new criteria for the oscillation of $n$th order nonlinear dynamic equations of the form

$$
x^{\Delta^{n}}(t)+q(t)\left(x^{\sigma}(\xi(t))\right)^{\lambda}=0
$$

are established in delay $\xi(t) \leq t$ and non-delay $\xi(t)=t$ cases, where $n \geq 2$ is a positive integer, $\lambda$ is the ratio of positive odd integers. Many of the results are new for the corresponding higher order difference equations and differential equations are as special cases.

Mathematics Subject Classification (2011): 34C10; 34C15.
\end{abstract}

Keywords: oscillation, neutral, time scale, higher order

\section{Introduction}

Consider the $n$th order nonlinear delay dynamic equation

$$
x^{\Delta^{n}}(t)+q(t)\left(x^{\sigma}(\xi(t))\right)^{\lambda}=0
$$

on an arbitrary time-scale $\mathbb{T} \subseteq \mathbb{R}$ with sup $\mathbb{T}=\infty$ and $0 \in \mathbb{T}$, where $n \geq 2$ is a positive integer, $\lambda$ is the ratio of positive odd integers, $q: \mathbb{T} \rightarrow \mathbb{R}^{+}=(0, \infty)$ and $\xi: \mathbb{T} \rightarrow \mathbb{T}$ are real-valued rd-continuous functions, $\xi(t) \leq t, \xi^{\Delta}(t) \geq 0$, and $\lim _{t \rightarrow \infty} \xi(t)=$ $\infty$. Throughout the article by $t \geq s$ for $t, s \in \mathbb{T}$ we shall mean $t \in[s, \infty) \cap \mathbb{T}:=[s, \infty)_{\mathbb{V}}$. For the forward jump operator $\sigma$, we use the usual notation $x^{\sigma}=x \circ \sigma$.

We recall that a solution $x$ of Equation (1.1) is said to be nonoscillatory if there exists a $t_{0} \in \mathbb{T}$ such that $x(t) x(\sigma(t))>0$ for all $t \geq t_{0}$; otherwise, it is said to be oscillatory. Equation (1.1) is said to be oscillatory if all its solutions are oscillatory.

Recently, there has been an increasing interest in studying the oscillatory behavior of first-and second-order dynamic equations on time-scales, see [1-7]. However, there are very few results regarding the oscillation of higher order equations. Therefore, the purpose of this article is to obtain new criteria for the oscillation of Equation (1.1). This topic is fairly new for dynamic equations on time scales. For a general background on time scale calculus, we may refer to $[8,9]$.

The article is organized as follows: In Section 2, some preliminary lemmas and notations are given, while Section 3 is devoted to the study of Equation (1.1) via comparison with a set of second-order dynamic equations whose oscillatory character is

(c) 2012 Grace et al; licensee Springer. This is an Open Access article distributed under the terms of the Creative Commons Attribution License (http://creativecommons.org/licenses/by/2.0), which permits unrestricted use, distribution, and reproduction in any medium, provided the original work is properly cited. 
known and have been investigated extensively in the literature. In Section 4, we establish new oscillation criteria for Equation (1.1) when $\xi(t)=t$ for linear, sublinear, and superlinear cases. Further results are presented in Section 5 when there is a special restriction on the function $q$. We should note that many of our results of this article are new for the corresponding higher order nonlinear differential and difference equations. In fact, the obtained results extend, unify and correlate many of the existing results in the literature.

\section{Preliminaries}

We shall employ the following lemmas. The first lemma is the well-known Kiguradze's lemma.

Lemma 2.1. Let $x \in C_{r d}^{m}\left(\left[t_{0}, \infty\right), \mathbb{R}^{+}\right)$. If $x^{\Delta^{m}}(t)$ is of constant sign on $\left[t_{0}, \infty\right)_{\mathbb{T}}$ and not identically zero on $\left[t_{1}, \infty\right)_{\mathbb{W}}$ for any $t_{1} \geq t_{0}$, then there exist $a t_{x} \geq t_{0}$ and an integer $\ell, 0 \leq \ell \leq m$ with $m+\ell$ even for $x^{\Delta^{m}}(t) \geq 0$, or $m+\ell$ odd for $x^{\Delta^{m}}(t) \leq 0$ such that

$$
\ell>0 \text { implies } x^{\Delta^{k}}(t)>0 \quad \text { for } t \geq t_{x}, \quad k \in\{1,2, \ldots, \ell-1\}
$$

and

$$
\ell \leq m-1 \text { implies }(-1)^{\ell+k} x^{\Delta^{k}}(t)>0 \text { for } t \geq t_{x}, k \in\{\ell, \ell+1, \ldots, m-1\} .
$$

Lemma 2.2. If the inequality

$$
x^{\Delta \Delta}+Q(t) x^{\lambda} \leq 0,
$$

where $Q$ is a positive real-valued, rd-continuous function on $\mathbb{T}$, has an eventually positive solution, then the equation

$$
x^{\Delta \Delta}+Q(t) x^{\lambda}=0
$$

also has an eventually positive solution.

Proof. Let $x(t)$ be an eventually positive solution of inequality (2.3). It is easy to see that $x^{\Delta}(t)>0$ eventually. Let $t_{0}$ be sufficiently large so that $x(t)>0$ and $y(t)=: x^{\Delta}(t)>$ 0 for $t \in\left[t_{0}, \infty\right)_{\mathbb{T}}$. Then in view of

$$
x(t)=x\left(t_{0}\right)+\int_{t_{0}}^{t} y(s) \Delta s,
$$

(2.3) becomes

$$
y^{\Delta}(t)+Q(t)\left(x\left(t_{0}\right)+\int_{t_{0}}^{t} y(s) \Delta s\right)^{\lambda} \leq 0, t \in\left[t_{0}, \infty\right)_{\mathbb{J}} .
$$

Integrating (2.5) from $t$ to $u \geq t \geq t_{0}$ and letting $u \rightarrow \infty$, we have

$$
y(t) \geq F(t, y(t)), \quad t \in\left[t_{0}, \infty\right)_{\mathbb{T}},
$$


where

$$
F(t, \gamma):=\int_{t}^{\infty} \mathrm{Q}(v)\left(x\left(t_{0}\right)+\int_{t_{0}}^{v} y(s) \Delta s\right)^{\lambda} \Delta v .
$$

Next, we define a sequence of successive approximations $\left\{z_{j}(t)\right\}$ as follows:

$$
\begin{aligned}
& z_{0}(t)=\gamma(t) \\
& z_{j+1}(t)=F\left(t, z_{j}(t)\right), \quad j=0,1,2, \ldots .
\end{aligned}
$$

It is easy to show that

$$
0<z_{j}(t) \leq y(t) \text { and } z_{j+1}(t) \leq z_{j}(t), \quad j=0,1,2, \ldots
$$

Thus the sequence $\left\{z_{j}(t)\right\}$ is nonincreasing and bounded for each $t \geq t_{0}$. This means we may define $z(t)=\lim _{j \rightarrow \infty} z_{j}(t) \geq 0$. Since $0 \leq z(t) \leq z_{j}(t) \leq y(t)$ for all $j \geq 0$, we find that

$$
\int_{t_{0}}^{t} z_{j}(s) \Delta s \leq \int_{t_{0}}^{t} \gamma(s) \Delta s .
$$

By the Lebesgue dominated convergence theorem on time scales, one can easily obtain

$$
z(t)=F(t, z(t)) .
$$

Therefore,

$$
z^{\Delta}(t)=-Q(t) m^{\lambda}(t),
$$

where

$$
m(t)=x\left(t_{0}\right)+\int_{t_{0}}^{t} z(s) \Delta s .
$$

Then, $m(t)>0$ and $m^{\Delta}(t)=z(t)$. Equation (2.6) then gives

$$
m^{\Delta \Delta}(t)+Q(t) m^{\lambda}(t)=0 .
$$

Hence, Equation (2.4) has a positive solution $m(t)$. This completes the proof. $\square$

Lemma 2.3 ([4]). Suppose $|x|^{\Delta}$ is of one sign on $\left[t_{0}, \infty\right)_{\mathbb{V}}$ and $\alpha>0, \alpha \neq 1$. Then

$$
\frac{|x|^{\Delta}}{\left(\left|x^{\sigma}\right|\right)^{\alpha}} \leq \frac{\left(|x|^{1-\alpha}\right)^{\Delta}}{(1-\alpha)} \leq \frac{|x|^{\Delta}}{\left(|x|^{\alpha}\right)}, \quad t \geq t_{0} .
$$

It will be convenient to employ the Taylor monomials (see [[8], Sect. 1.6]) $n \in \mathbb{N}_{0}, n \in \mathbb{N}_{0}$, which are defined recursively as follows:

$$
h_{0}(t, s)=g_{0}(t, s)=1 \text {, }
$$




$$
h_{n+1}(t, s)=\int_{s}^{t} h_{n}(\tau, s) \Delta \tau, \quad g_{n+1}(t, s)=\int_{s}^{t} g_{n}(\sigma(\tau), s) \Delta \tau, \quad t, s \in \mathbb{T}, n \in \mathbb{N}_{0} .
$$

It is clear that $h_{1}(t, s)=g_{1}(t, s)=t-s$ for any time-scales, but simple formulas in general do not hold for $n \geq 2$. It is also known that

$$
h_{n}(t, s)=(-1)^{n} g_{n}(s, t) \text {. }
$$

\section{Comparison criteria for delay dynamic equations}

In this section, we shall consider the equation

$$
x^{\Delta^{n}}(t)+q(t) x^{\lambda}(\xi(t))=0 .
$$

For $t_{0} \in \mathbb{T}$ and $\ell \in\{1,2, \ldots, n-1\}$, we define

$$
q_{\ell}\left(t, t_{0}\right)=\int_{t}^{\infty} \tau^{-\lambda} Q_{\ell}\left(\tau, t, t_{0}\right) \Delta \tau, \quad t \in\left[t_{0}, \infty\right)_{\mathbb{T}},
$$

where

$$
Q_{\ell}\left(\tau, t, t_{0}\right)=g_{n-\ell-2}(\sigma(\tau), t) R_{\ell}^{\lambda}\left(\tau, t_{0}\right) q(\tau), \quad \tau \geq t .
$$

with

$$
R_{\ell}\left(\tau, t_{0}\right)= \begin{cases}\int_{t_{0}}^{\xi(\tau)} s h_{\ell-2}(\xi(\tau), \sigma(s) \Delta s, & \ell \geq 2 \\ \xi(\tau), & \ell=1 .\end{cases}
$$

Theorem 3.1. Let $t_{0} \in \mathbb{T}$. Suppose that for every $\ell \in\{1,2, \ldots, n-1\}$,

$$
\int^{\infty} Q_{\ell}\left(\tau, t_{0}, t_{0}\right) \Delta \tau=\infty
$$

Then, Equation (3.1) is oscillatory if

(i) for $n$ even, the equation

$$
y^{\Delta \Delta}+q_{\ell}\left(t, t_{0}\right) y^{\lambda}=0
$$

for all $\ell \in\{1,3, \ldots, n-1\}$ is oscillatory;

(ii) for $n$ odd, the Equation (3.3) for all $\ell \in\{2,4, \ldots, n-1\}$ is oscillatory, and

$$
\limsup _{t \rightarrow \infty} \int_{\xi(t)}^{t} h_{n-1}^{\lambda}(\xi(s), \xi(t)) q(s) \Delta s>\left\{\begin{array}{l}
0 \text { when } 0<\lambda<1 \\
1 \text { when } \lambda=1 .
\end{array}\right.
$$

Proof. Let $x(t)$ be a nonoscillatory solution of Equation (3.1). Without loss of generality, we may assume that $x(t)>0$ and $x(\xi(t))>0$ for $t \geq t_{0}$, since otherwise the substitution $w=-x$ transforms Equation (3.1) into an equation of the same form subject to the assumptions of the theorem. 
By Lemma 2.1, there exist a $t_{1} \geq t_{0}$ and an integer $\ell \in\{0,1, \ldots, n\}$ with $n+\ell$ odd such that (2.1) and (2.2) hold for all $t \geq t_{1}$. We see that

$$
x^{\Delta^{\ell-1}}(t)>0, x^{\Delta^{\ell}}(t)>0, x^{\Delta^{\ell+1}}(t)<0 \quad \text { for } t \geq t_{1},
$$

and by Taylor's formula

$$
\begin{aligned}
x(t) & =\sum_{k=0}^{l-2} x^{\Delta^{k}}\left(t_{1}\right) h_{k}\left(t, t_{1}\right)+\int_{t_{1}}^{t} h_{\ell-2}(t, \sigma(\tau)) x^{\Delta^{\ell-1}}(\tau) \Delta \tau \\
& \geq \int_{t_{1}}^{t} h_{\ell-2}(t, \sigma(\tau)) x^{\Delta^{\ell-1}}(\tau) \Delta \tau \quad \text { for } \ell>1 .
\end{aligned}
$$

We claim that

$$
\frac{x^{\Delta^{\ell-1}(t)}}{t} \text { is strictly decreasing for } t \geq t_{1} \text { and } \ell>0 .
$$

To prove it, set $X(t)=x^{\Delta^{\ell-1}}(t)-t x^{\Delta^{\ell}}(t)$. Because

$$
\left(\frac{x^{\Delta^{\ell-1}}}{t}\right)^{\Delta}=\frac{t x^{\Delta^{\ell}}-x^{\Delta^{\ell-1}}}{t \sigma(t)}=-\frac{X(t)}{t \sigma(t)},
$$

it suffices to show that $X(t)$ is strictly positive. Suppose on the contrary that $X(t)<0$. Then $x^{\Delta^{\ell-1}} / t$ is strictly increasing and hence

$$
x^{\Delta^{\ell-1}}(t) \geq c t \quad \text { for } t \geq t_{1}
$$

where $c=x^{\Delta^{\ell-1}}\left(t_{1}\right) / t_{1}>0$. Using (3.7) in (3.5), we have

$$
x(\xi(t)) \geq c \int_{t_{1}}^{\xi(t)} \tau h_{\ell-2}(\xi(t), \sigma(\tau)) \Delta \tau .
$$

Let $\ell=1$, then (3.7) gives $x(\xi(t)) \geq c \xi(t)$ for $t \geq t_{1}$ by increasing the size of $t_{1}$ if necessary. Thus, we obtain

$$
x(\xi(t)) \geq c R_{\ell}\left(t, t_{1}\right) \quad \text { for } t \geq t_{1} \text { and } \ell>0 .
$$

On the other hand, by Taylor's formula we may write that

$$
\begin{aligned}
x^{\Delta^{t+1}}(t) & =\sum_{k=0}^{n-\ell-2} x^{\Delta^{\ell+k+1}}(s) h_{k}(t, s)+\int_{t}^{s} h_{n-\ell-2}(t, \sigma(\tau))\left(-x^{\Delta^{n}}(\tau)\right) \Delta \tau \\
& =\sum_{k=0}^{n-\ell-2} x^{\Delta^{\ell+k+1}}(s)(-1)^{k} g_{k}(s, t)+\int_{t}^{s}(-1)^{n-\ell-2} g_{n-\ell-2}(\sigma(\tau), t)\left(-x^{\Delta^{n}}(\tau)\right) \Delta \tau \\
& \leq-\int_{t}^{\infty} g_{n-\ell-2}(\sigma(\tau), t) q(\tau) x^{\lambda}(\xi(\tau)) \Delta \tau .
\end{aligned}
$$


From (3.9) and (3.10), we have

$$
-x^{\Delta^{\ell+1}}\left(t_{1}\right) \geq c^{\lambda} \int_{t_{1}}^{\infty} g_{n-\ell-2}\left(\sigma(\tau), t_{1}\right) q(\tau) R_{\ell}^{\lambda}\left(\tau, t_{1}\right) \Delta \tau,
$$

which contradicts (3.2), and hence completes the proof of the claim.

Now in view of (3.6) it follows from (3.5) that

$$
x(t) \geq \frac{x^{\Delta^{\ell-1}}(t)}{t} \int_{t_{1}}^{t} \tau h_{\ell-2}(t, \sigma(\tau)) \Delta \tau, \quad t \geq t_{1} .
$$

Replacing $t$ by $\xi(t)$ in (3.12) and using (3.6), we have

$$
x(\xi(t)) \geq x^{\Delta^{\ell-1}}(t) \int_{t_{1}}^{\xi(t)} \frac{\tau}{t} h_{\ell-2}(\xi(t), \sigma(\tau)) \Delta \tau, \quad \ell>1
$$

for all $t \geq t_{2}$ for some $t_{2} \geq t_{1}$.

If $\ell=1$, then we may write that

$$
x(\xi(t))=\frac{x^{\Delta^{\ell-1}}(\xi(t))}{\xi(t)} \xi(t) \geq \frac{x^{\Delta^{\ell-1}}(t)}{t} \xi(t), \quad t \geq t_{2}
$$

Thus, from (3.13) and (3.14) for all $t \geq t_{2}$,

$$
x(\xi(t)) \geq \frac{x^{\Delta^{\ell-1}}(t)}{t} R_{\ell}\left(t, t_{1}\right), \quad \ell>0 .
$$

Substituting (3.15) into (3.10) gives

$$
-x^{\Delta^{\ell+1}}(t) \geq\left(x^{\Delta^{\ell-1}}(t)\right)^{\lambda} \int_{t}^{\infty} \tau^{-\lambda} g_{n-\ell-2}(\sigma(\tau), t) R_{\ell}^{\lambda}\left(\tau, t_{1}\right) q(\tau) \Delta \tau, t \geq t_{2} .
$$

Set $w(t)=x^{\Delta^{\ell-1}}(t)$ in (3.16), then $w(t)>0$ satisfies

$$
w^{\Delta \Delta}+q_{\ell}\left(t, t_{1}\right) w^{\lambda} \leq 0, \quad t \geq t_{2} .
$$

By Lemma 2.2, the equation

$$
w^{\Delta \Delta}+q_{\ell}\left(t, t_{1}\right) w^{\lambda}=0
$$

has a nonoscillatory solution. But this is impossible by the hypothesis.

Finally, we let $\ell=0$. This is the case, when $n$ is odd. By applying Taylor's formula and using (2.2) with $\ell=0$, we can easily find

$$
x(u) \geq h_{n-1}(u, v) x^{\Delta^{n-1}}(v)
$$

for $v \geq u \geq t_{1}$, which implies that

$$
x(\xi(s)) \geq h_{n-1}(\xi(s), \xi(t)) x^{\Delta^{n-1}}(\xi(t)), \quad t>s \geq t_{3} .
$$


for some $t_{3} \geq t_{1}$. Integrating equation (3.1) from $\xi(t) \geq t_{3}$ to $t \geq t$, we get

$$
x^{\Delta^{n-1}}(\xi(t)) \geq \int_{\xi(t)}^{t} q(s) x^{\lambda}(\xi(s)) \Delta s
$$

Using (3.18) in (3.19), we have

$$
x^{\Delta^{n-1}}(\xi(t)) \geq\left(x^{\Delta^{n-1}}(\xi(t))\right)^{\lambda} \int_{\xi(t)}^{t} h_{n-1}^{\lambda}(\xi(s), \xi(t)) q(s) \Delta s
$$

or

$$
\left(x^{\Delta^{n-1}}(\xi(t))\right)^{1-\lambda} \geq \int_{\xi(t)}^{t} h_{n-1}^{\lambda}(\xi(s), \xi(t)) q(s) \Delta s
$$

Taking the lim sup as $t \rightarrow \infty$, we obtain a contradiction to condition (3.4).

The following immediate result can be extracted from Theorem 3.1.

Corollary 3.1. Let $n$ be an odd and condition (3.4) hold. Then every bounded solution of Equation (3.1) is oscillatory.

Next, we claim that inequality (3.15) can be replaced by

$$
x(\xi(t)) \geq \frac{1}{t} h_{\ell}\left(\xi(t), t_{1}\right) x^{\Delta^{\ell-1}}(t) .
$$

To prove this, we write that

$$
x^{\Delta^{\ell-2}}(t) \geq \int_{t_{1}}^{t} x^{\Delta^{\ell-1}}(s) \Delta s=\int_{t_{1}}^{t} s\left(\frac{x^{\Delta^{\ell-1}}(s)}{s}\right) \Delta s
$$

and hence by (3.6) we find

$$
x^{\Delta^{\ell-2}}(t) \geq h_{2}\left(t, t_{1}\right)\left(\frac{x^{\Delta^{\ell-1}}(t)}{t}\right) .
$$

Integrating this inequality $(\ell-2)$-times from $t_{1}$ to $t \geq t_{1}$ and using (3.6), we obtain

$$
x(t) \geq h_{\ell}\left(t, t_{1}\right)\left(\frac{x^{\Delta^{\ell-1}}(t)}{t}\right) .
$$

Thus, there exists a $t_{2} \geq t_{1}$ such that

$$
x(\xi(t)) \geq h_{\ell}\left(\xi(t), t_{1}\right) \frac{x^{\Delta^{\ell-1}}(\xi(t)}{\xi(t))} \geq \frac{1}{t} h_{\ell}\left(\xi(t), t_{1}\right) x^{\Delta^{\ell-1}}(t), \quad t \geq t_{2} .
$$

This completes the proof of our claim.

Set

$$
Q_{\ell}^{*}\left(\tau, t, t_{0}\right)=g_{n-\ell-2}(\sigma(\tau), t) h_{l}^{\lambda}\left(\xi(\tau), t_{0}\right) q(\tau), \quad \tau \geq t
$$


and

$$
q_{\ell}^{*}\left(t, t_{0}\right)=\int_{t}^{\infty} \tau^{-\lambda} Q_{\ell}^{*}\left(\tau, t, t_{0}\right) \Delta \tau, \quad t \geq t_{0}
$$

In view of Theorem 3.1 and inequality (3.20) we may state the following theorem.

Theorem 3.2. In Theorem 3.1, let $q_{\ell}$ and $Q_{\ell}$ be replaced by $q_{\ell}^{*}$ and $Q_{\ell}^{*}$, respectively. Then the conclusions of Theorem 3.1 hold.

Let $\mathbb{T}=\mathbb{R}$, i.e., the continuous case. Here Equation (3.1) becomes

$$
x^{(n)}(t)+q(t) x^{\lambda}(\xi(t))=0
$$

and the functions $q_{\ell}^{*}$ and $Q_{\ell}^{*}$ take the form

$$
q_{\ell}^{c}\left(t, t_{0}\right)=\int_{t}^{\infty} \tau^{-\lambda} Q_{\ell}^{c}\left(\tau, t, t_{0}\right) d \tau
$$

and

$$
Q_{\ell}^{c}\left(\tau, t, t_{0}\right)=\frac{\left(\xi(\tau)-t_{0}\right)^{\lambda \ell}}{(\ell !)^{\lambda}} \frac{(\tau-t)^{n-\ell-2}}{(n-\ell-2) !} q(\tau) .
$$

From Theorem 3.2 we have the following theorem.

Theorem 3.3. Let $t_{0} \in \mathbb{T}$. Suppose that for $\ell \in\{1,2, \ldots, n-1\}$,

$$
\int^{\infty} Q_{\ell}^{c}\left(\tau, t_{0}, t_{0}\right) d \tau=\infty
$$

Then, Equation (3.21) is oscillatory if

(i) for $n$ even, the equation

$$
\gamma^{\prime \prime}+q_{\ell}^{c}\left(t, t_{0}\right) y=0
$$

for all $\ell \in\{1,3, \ldots, n-1\}$ is oscillatory;

(ii) for $n$ odd, the Equation (3.23) for all $\ell \in\{2,4, \ldots, n-1\}$ is oscillatory and

$$
\limsup _{t \rightarrow \infty} \int_{\xi(t)}^{t}\left(\frac{(\xi(s)-\xi(t))^{n-1}}{(n-1) !}\right)^{\lambda} q(s) \Delta s>\left\{\begin{array}{l}
0 \text { when } 0<\lambda<1 \\
1 \text { when } \lambda=1 .
\end{array}\right.
$$

Next, we let $\mathbb{T}=\mathbb{Z}$, i.e., the discrete case. Then, Equation (3.1) reads as

$$
\Delta^{n} x(m)+q(m) x^{\lambda}(\xi(m)=0
$$

and the functions $q_{\ell}^{*}$ and $Q_{\ell}^{*}$ become

$$
q_{\ell}^{d}\left(m, m_{0}\right)=\sum_{j=m}^{\infty} j^{-\lambda} Q_{\ell}^{d}\left(j, m, m_{0}\right)
$$


and

$$
Q_{\ell}^{d}\left(j, m, m_{0}\right)=\frac{\left[\left(\xi(j)-m_{0}\right)^{(\ell)}\right]^{\lambda}}{(\ell !)^{\lambda}} \frac{(j-m+n-\ell-2)^{(n-\ell-2)}}{(n-\ell-2) !} q(j),
$$

where $t^{(m)}=t(t-1)(t-2) \ldots(t-m+1)$ is the usual factorial function.

Theorem 3.4. Let $m_{0} \in \mathbb{Z}$. Suppose that for $\ell \in\{1,2, \ldots, n-1\}$

$$
\sum_{j=m_{0}}^{\infty} Q_{\ell}^{d}\left(j, m_{0}, m_{0}\right)=\infty
$$

Then, Equation (3.25) is oscillatory if

(i) for $n$ even, the second-order difference equation

$$
\Delta^{2} y(m)+q_{\ell}^{d}\left(m, m_{0}\right) \gamma^{\lambda}(m)=0,
$$

for all $\ell \in\{1,3, \ldots, n-1\}$ is oscillatory;

(ii) for $n$ odd, the Equation (3.27) for all $\ell \in\{2,4, \ldots, n-1\}$ is oscillatory and

$$
\limsup _{m \rightarrow \infty} \sum_{j=\xi(m)}^{m}\left(\frac{(\xi(j)-\xi(m))^{(n-1)}}{(n-1) !}\right)^{\lambda} q(j)>\left\{\begin{array}{l}
0 \text { when } 0<\lambda<1 \\
1 \text { when } \lambda=1 .
\end{array}\right.
$$

Remark 1. The oscillation of Equation (3.1) is obtained via a comparison with a set of second-order dynamic equations whose oscillatory behavior has been studied extensively in the literature. In fact, there are many sufficient conditions for the oscillation of Equation (3.3) which can be employed rather easily.

\section{Even order dynamic equations without delay}

In this section, we present new oscillation criteria for (3.1) when $n$ is even. That is, we consider

$$
x^{\Delta^{2 n}}+q(t)\left(x^{\sigma}\right)^{\lambda}=0 .
$$

For $t \in \mathbb{T}$, we define

$$
\hat{Q}_{\ell}(t)=\int_{t}^{\infty} \int_{s_{2 n-\ell-1}}^{\infty} \ldots \int_{s_{1}}^{\infty} q(s) \Delta s \Delta s_{1} \ldots \Delta s_{2 n-\ell-1}, \ell \in\{1,3, \ldots, 2 n-1\} .
$$

Theorem 4.1. Let $\lambda>1$ and $t_{0} \in \mathbb{T}$. If for every integer $\ell \in\{1,3, \ldots, 2 n-1\}$,

$$
\int_{t_{0}}^{\infty} h_{\ell-1}\left(s, t_{0}\right) \hat{Q}_{\ell}(s) \Delta s=\infty
$$

then Equation (4.1) is oscillatory.

Proof. Let $x(t)$ be a nonoscillatory solution of Equation (4.1), say, $x(t)>0$ for $t \geq t_{0}$. From Equation (4.1), we see that $x^{\Delta^{2 n}}(t) \leq 0$ for $t \geq t_{0}$, where $x^{\Delta^{2 n}}(t)$ is not identically zero for all large $t$. Using Lemma 2.1 there exist a $t_{1} \geq t_{0}$ and an integer $\ell \in\{1,3$, $\ldots, 2 n-1\}$ such that (2.1) and (2.2) hold for all $t \geq t_{1}$. From (2.1), we see that $x^{\Delta^{\ell}}(t)>0$ and decreasing on $\left[t_{1}, \infty\right)_{\mathbb{T}}$. Now, 


$$
x^{\Delta^{\ell-1}}(s)-x^{\Delta^{\ell-1}}\left(t_{1}\right)=\int_{t_{1}}^{s} x^{\Delta^{\ell}}(\tau) \Delta \tau \geq h_{1}\left(s, t_{1}\right) x^{\Delta^{\ell}}(s),
$$

or

$$
x^{\Delta^{\ell-1}}(s) \geq h_{1}\left(s, t_{1}\right) x^{\Delta^{\ell}}(s), \quad s \geq t_{1} .
$$

Integrating (4.4) $(\ell-2)$-times from $t_{1}$ to $s \geq t_{1}$, we have

$$
x^{\Delta}(s) \geq h_{\ell-1}\left(s, t_{1}\right) x^{\Delta^{\ell}}(s), \quad s \geq t_{1} .
$$

Next, we integrate Equation (4.1) from $s_{1} \geq t_{1}$ to $v \geq s_{1}$ and let $v \rightarrow \infty$ to get

$$
x^{\Delta^{2 n-1}}\left(s_{1}\right) \geq \int_{s_{1}}^{\infty} q(\tau) x^{\lambda}(\sigma(\tau)) \Delta \tau \geq\left(\int_{s_{1}}^{\infty} q(\tau) \Delta \tau\right) x^{\lambda}\left(\sigma\left(s_{1}\right)\right) .
$$

Integrating this inequality from $s_{2} \geq t_{1}$ to $v \geq s_{2}$ and then letting $v \rightarrow \infty$ and using (2.2), we get

$$
-x^{\Delta^{2 n-2}}\left(s_{2}\right) \geq\left(\int_{s_{2}}^{\infty} \int_{s_{1}}^{\infty} q(\tau) \Delta \tau \Delta s_{1}\right) x^{\lambda}\left(\sigma\left(s_{2}\right)\right) .
$$

Continuing this process, one can easily find

$$
x^{\Delta^{\ell}}(s) \geq\left(\int_{s}^{\infty} \int_{s_{2 n-\ell-1}}^{\infty} \ldots \int_{s_{1}}^{\infty} q(\tau) \Delta \tau \Delta s_{1} \ldots \Delta s_{2 n-\ell-1}\right) x^{\lambda}(\sigma(s)),
$$

or

$$
x^{\Delta^{\ell}}(s) \geq \hat{Q}_{\ell}(s) x^{\lambda}(\sigma(s)), \quad s \geq t_{1} .
$$

From (4.5) and (4.6), we find

$$
x^{-\lambda}(\sigma(s)) x^{\Delta}(s) \geq h_{\ell-1}\left(s, t_{1}\right) \hat{Q}_{\ell}(s), \quad s \geq t_{1},
$$

and hence

$$
\int_{t_{1}}^{t} x^{-\lambda}(\sigma(s)) x^{\Delta}(s) \Delta s \geq \int_{t_{1}}^{t} h_{\ell-1}\left(s, t_{1}\right) \hat{Q}_{\ell}(s) \Delta s .
$$

By employing the first inequality in Lemma 2.3, we get

$$
\int_{t_{1}}^{t} \frac{\left(x^{1-\lambda}(s)\right)^{\Delta}}{1-\lambda} \Delta s \geq \int_{t_{1}}^{t} h_{\ell-1}\left(s, t_{1}\right) \hat{Q}_{\ell}(s) \Delta s
$$

and so

$$
\int_{t_{1}}^{\infty} h_{\ell-1}\left(s, t_{1}\right) \hat{Q}_{\ell}(s) \Delta s \leq \frac{x^{1-\lambda}\left(t_{1}\right)}{\lambda-1}<\infty .
$$


But this contradicts condition (4.3). The proof is complete.

Theorem 4.2. Let $\lambda>1$ and $t_{0} \in \mathbb{T}$. If for every integer $\ell \in\{1,3, \ldots, 2 n-1\}$,

$$
\int_{t_{0}}^{\infty} h_{\ell-1}\left(s, t_{0}\right)\left(\int_{s}^{\infty} g_{2 n-\ell-1}(\sigma(\tau), s) q(\tau) \Delta \tau\right) \Delta s=\infty,
$$

then Equation (4.1) is oscillatory.

Proof. Let $x(t)$ be a nonoscillatory solution of Equation (1.1), say, $x(t)>0$ for $t \geq t_{0}$. By Taylor's formula, we see that

$$
x^{\Delta^{\ell}}(s) \geq-\int_{s}^{\infty} g_{2 n-\ell-1}(\sigma(\tau), s) x^{\Delta^{2 n}}(\tau) \Delta \tau, \quad s \geq t_{1} .
$$

Using Equation (4.1) in (4.8), we get

$$
\begin{aligned}
x^{\Delta^{\ell}}(s) & \geq \int_{s}^{\infty} g_{2 n-\ell-1}(\sigma(\tau), s) q(\tau) x^{\lambda}(\sigma(\tau)) \Delta \tau \\
& \geq\left(\int_{s}^{\infty} g_{2 n-\ell-1}(\sigma(\tau), s) q(\tau) \Delta \tau\right) x^{\lambda}(\sigma(s)), \quad s \geq t_{1} .
\end{aligned}
$$

Combining (4.8) with (4.9), we find

$$
x^{\Delta}(s) \geq h_{\ell-1}\left(s, t_{1}\right)\left(\int_{s}^{\infty} g_{2 n-\ell-1}(\sigma(\tau), s) q(\tau) \Delta \tau\right) x^{\lambda}(\sigma(s)), \quad s \geq t_{1} .
$$

Dividing both sides by $x^{\lambda}(\sigma(s))$ and integrating from $t_{1}$ to $t \geq t_{1}$, we have

$$
\int_{t_{1}}^{t} x^{-\lambda}(\sigma(s)) x^{\Delta}(s) \Delta s \geq \int_{t_{1}}^{t} h_{\ell-1}\left(s, t_{1}\right) \int_{s}^{\infty} g_{2 n-\ell-1}(\sigma(\tau), s) q(\tau) \Delta \tau \Delta s .
$$

The rest of the proof is similar to that of Theorem 4.1 and hence it is omitted. This completes the proof.

Next, we apply Theorems 4.1 and 4.2 to obtain oscillation criteria for Equation (4.1) when $\lambda \leq 1$.

Theorem 4.3. Let $\lambda \leq 1$ and $t_{0} \in \mathbb{T}$. Assume that there exists a positive constant $\alpha$ such that $\alpha+\lambda>1$. If for every $\ell \in\{1,3, \ldots, 2 n-1\}$, condition (4.3) or (4.7) holds with $q(t)$ replaced by $c q(t) h_{\ell}^{-\alpha}(t, 0)$, where $c$ is any positive constant, then Equation (4.1) is oscillatory.

Proof. Let $x(t)$ be a nonoscillatory solution of Equation (4.1) and assume that there exists a $t_{0}>0$ such that $x(t)>0$ for $t \geq t_{0}$ and (2.1) and (2.2) hold for $t \geq t_{0}$. From (2.1) and the decreasing nature of $x^{\Delta^{\ell}}(t)$, there exists a constant $c_{1}>0$ such that $x^{\Delta^{\ell}}(t) \leq c_{1}$ for $t \geq t_{0}$. Integrating this inequality $\ell$-times from $t_{0}$ to $t$, we have

$$
x(t) \leq \operatorname{ch}_{\ell}(t, 0), \quad t \geq t_{0}
$$


where $c$ is a positive constant. Now, from Equation (4.1), we have

$$
\begin{aligned}
0 & =x^{\Delta^{2 n}}(t)+q(t) x^{-\alpha}(\sigma(t)) x^{\lambda+\alpha}(\sigma(t)) \\
& \geq x^{\Delta^{2 n}}(t)+c^{-\alpha} q(t) h_{\ell}^{-\alpha}(\sigma(t), 0) x^{\lambda+\alpha}(\sigma(t)), \quad t \geq t_{0} .
\end{aligned}
$$

By applying Theorems 4.1 and 4.2 with inequality (3.20), we arrive at the desired conclusion. This completes the proof. $\square$

Theorem 4.4. Let $\lambda<1$ and $t_{0} \in \mathbb{T}$. If for every $\ell \in\{1,3, \ldots, 2 n-1\}$,

$$
\int_{t_{0}}^{\infty} q(t)\left(\int_{t_{0}}^{t} h_{\ell-1}(t, \sigma(u)) g_{2 n-\ell-1}(t, u) \Delta u\right)^{\lambda} \Delta t=\infty
$$

then Equation (4.1) is oscillatory.

Proof. Let $x(t)$ be a nonoscillatory solution of Equation (4.1), say, $x(t)>0$ for $t \geq t_{0}$. As in the proof of Theorem 4.1, we see that (2.1) and (2.2) hold for $t \geq t_{1} \geq t_{0}$. It is easy to see that

$$
x(t) \geq \int_{t_{1}}^{t} h_{\ell-1}(t, \sigma(u)) x^{\Delta^{\ell}}(u) \Delta u
$$

and

$$
x^{\Delta^{\ell}}(u) \geq g_{2 n-\ell-1}(t, u) x^{\Delta^{2 n-1}}(t), \quad t \geq u \geq t_{1} .
$$

Therefore,

$$
x(t) \geq\left(\int_{t_{1}}^{t} h_{\ell-1}(t, \sigma(u)) g_{2 n-\ell-1}(t, u) \Delta u\right) x^{\Delta^{2 n-1}}(t) \text { for } t \geq t_{1} .
$$

Using this inequality in Equation (4.1), we get

$$
\begin{aligned}
-\left(x^{\Delta^{2 n-1}}(t)\right)^{\Delta} & =q(t) x^{\lambda}(\sigma(t)) \geq q(t) x^{\lambda}(t) \\
& \geq q(t)\left(\int_{t_{1}}^{t} h_{\ell-1}(t, \sigma(u)) g_{2 n-\ell-1}(t, u) \Delta u\right)^{\lambda}\left(x^{\Delta^{2 n-1}}(t)\right)^{\lambda}, \quad t \geq t_{1} .
\end{aligned}
$$

Set $w(t)=x^{\Delta^{2 n-1}}(t)$, then

$$
-w^{\lambda}(t) w^{\Delta}(t) \geq q(t)\left(\int_{t_{1}}^{t} h_{\ell-1}(t, \sigma(u)) g_{2 n-\ell-1}(t, u) \Delta u\right)^{\lambda}, \quad t \geq t_{1} .
$$

Finally, in view of a chain rule, we integrate the last inequality from $t_{1}$ to $t$ to get

$$
\infty>\frac{w^{1-\lambda}\left(t_{1}\right)}{1-\lambda} \geq \int_{t_{1}}^{t} q(s)\left(\int_{t_{1}}^{s} h_{\ell-1}(s, \sigma(u)) g_{2 n-\ell-1}(s, u) \Delta u\right)^{\lambda} \Delta s,
$$

a contradiction with condition (4.12). $\square$ 
As an example, we shall reformulate some of the above results for the case $\mathbb{T}=\mathbb{Z}$, i. e., the discrete case. The Equation (4.1) takes the form

$$
\Delta^{2 n} x(m)+q(t) x^{\lambda}(m+1)=0
$$

and establish new criteria for the oscillation of Equation (4.13).

We let

$$
\hat{Q}_{\ell}^{d}(m)=\sum_{s_{2 n-\ell-1}=t}^{\infty} \cdots \sum_{s_{1}=s_{2}}^{\infty} \sum_{u=s_{1}}^{\infty} q(u), \quad \ell \in\{1,3, \ldots, 2 n--1\}, \quad m \geq m_{0} .
$$

Theorem 4.5. Let $\lambda>1$ and $m_{0} \in \mathbb{Z}$. If for every $\ell \in\{1,3, \ldots, 2 n-1\}$,

$$
\sum_{s=m_{0}}^{\infty} s^{(\ell-1)} \hat{Q}_{\ell}^{d}(s)=\infty
$$

then Equation (4.13) is oscillatory.

Theorem 4.6. Let $\lambda>1$ and $m_{0} \in \mathbb{Z}$. If for every $\ell \in\{1,3, \ldots, 2 n-1\}$,

$$
\sum_{s=m_{0}}^{\infty} s^{(\ell-1)} \sum_{\tau=s}^{\infty}(\tau-s+1)^{(2 n-\ell-1)} q(\tau)=\infty,
$$

then Equation (4.13) is oscillatory.

Theorem 4.7. Let $\lambda<1$ and $m_{0} \in \mathbb{Z}$. If

$$
\sum_{s=m_{0}}^{\infty}\left(s^{\lambda}\right)^{(2 n-1)} q(s)=\infty,
$$

then Equation (4.13) is oscillatory.

Theorem 4.8. Let $\lambda \leq 1$ and $m_{0} \in \mathbb{Z}$. Assume that there exists a positive constant $\alpha$ such that $\alpha+\lambda>1$. If for every $\ell \in\{1,3, \ldots, 2 n-1\}$ condition (4.14) or (4.15) holds with $q(t)$ be replaced by $\left.c q(t)(\mathrm{t})^{(\ell)} / \ell !\right)^{-\alpha}$, where $c$ is any positive constant, then Equation (4.13) is oscillatory.

Remark 2. For Equation (4.1) of odd order, one may obtain results for the oscillatory and asymptotic behavior, while for complete oscillation, we may consider Equation (1.1) and employ the technique given in Theorem 3.1. The details are left to the reader.

\section{Further oscillation criteria}

In this section, we consider

$$
x^{\Delta^{n}}+q(t)\left(x^{\sigma}\right)^{\lambda}=0
$$

subject to the condition

$$
\int_{t_{0}}^{\infty} \int_{v}^{\infty} \int_{u}^{\infty} q(s) \Delta s \Delta u \Delta v=\infty .
$$

Note that if $x(t), t \geq t_{0}$ is a positive solution of Equation (5.1), then by Lemma 2.1, Equations (2.1), and (2.2) hold for $t \geq t_{1}$. Here, we claim that $\ell=n-1$. Otherwise, we find $x^{\Delta^{n-1}}(t)>0, x^{\Delta^{n-2}}(t)<0$ and $x^{\Delta^{n-3}}(t)>0$ on $\left[t_{1}, \infty\right)_{\mathbb{T}}$. Integrating Equation 
(5.1) from $t \geq t_{1}$ to $u \geq t$ and letting $u \rightarrow \infty$, we have

$$
x^{\Delta^{n-1}}(t) \geq \int_{t}^{\infty} q(s) x^{\lambda}(\sigma(s)) \Delta s .
$$

Since $x$ is increasing on $\left[t_{1}, \infty\right)_{\mathbb{T}}$, there exists a constant $c>0$ such that

$$
x(t) \geq c, \quad t \in\left[t_{1}, \infty\right)_{\mathbb{T}} .
$$

Using (5.4) in (5.3), we get

$$
-x^{\Delta^{n-1}}(t) \leq-c^{\lambda} \int_{t}^{\infty} q(s) \Delta s
$$

Integrating this inequality twice, once from $v \geq t$ to $w \geq v$ and letting $w \rightarrow \infty$ and then from $t_{1}$ to $t \geq t_{1}$, we have

$$
x^{\Delta^{n-3}}(t) \leq-c^{\lambda} \int_{t_{1}}^{t} \int_{v}^{\infty} \int_{s}^{\infty} q(u) \Delta u \Delta s \Delta v \rightarrow \infty \quad \text { as } t \rightarrow \infty,
$$

which contradicts (5.2). Thus, we must have $\ell=n-1$, i.e.,

Thus, we have

$$
x^{\Delta^{n-2}}(t)=x^{\Delta^{n-2}}\left(t_{1}\right)+\int_{t_{1}}^{t} x^{\Delta^{n-1}}(s) \Delta s \geq h_{1}\left(t, t_{1}\right) x^{\Delta^{n-1}}(t), \quad t \geq t_{1} .
$$

Integrating this inequality $(n-2)$-times from $t_{1}$ to $t$, we obtain

$$
x(t) \geq h_{n-1}\left(t, t_{1}\right) x^{\Delta^{n-1}}(t), \quad t \geq t_{1} .
$$

Now, by making use of earlier results in [6], we obtain the following interesting theorems.

Theorem 5.1. Let condition (5.2) hold. If there exists a positive nondecreasing, differentiable function $\eta \in C_{r d}\left(\mathbb{T}, \mathbb{R}^{+}\right)$such that for any $t_{1} \geq t_{0}$,

$$
\underset{t \rightarrow \infty}{\limsup } \int_{t_{1}}^{t}\left[\eta(s) q(s)-\eta^{\Delta}(s) \frac{A\left(s, t_{0}\right)}{h_{n-1}\left(s, t_{0}\right)}\right] \Delta s=\infty
$$

where

$$
A\left(t, t_{0}\right)= \begin{cases}c_{1}, c_{1} \text { is any positive constant, } & \text { when } \lambda>1 \\ 1, & \text { when } \lambda=1 \\ c_{2} h_{n-1}^{1-\lambda}\left(t, t_{0}\right), c_{2} \text { is any positive constant, } & \text { when } \lambda<1,\end{cases}
$$

then Equation (5.1) is oscillatory.

Proof. Let $x(t)$ be a nonoscillatory solution of Equation (5.1), say, $x(t)>0$ for $t \geq t_{1} \geq$ $t_{0}$ 
Define

$$
w(t)=\eta(t) \frac{x^{\Delta^{n-1}}(t)}{x^{\lambda}(t)}, \quad t \geq t_{1}
$$

It is easy to see that for $t \geq t_{1}$,

$$
\begin{aligned}
w^{\Delta} & =\left(\frac{\eta}{x^{\lambda}}\right)^{\Delta}\left(x^{\Delta^{n-1}}\right)^{\sigma}+\left(\frac{\eta}{x^{\lambda}}\right)\left(x^{\Delta^{n}}\right) \\
& =-\eta q\left(\frac{x^{\sigma}}{x}\right)^{\lambda}+\left(x^{\Delta^{n-1}}\right)^{\sigma}\left[\frac{\eta^{\Delta} x^{\lambda}-\eta\left(x^{\lambda}\right) \Delta}{x^{\lambda}\left(x^{\sigma}\right)^{\lambda}}\right] .
\end{aligned}
$$

By [[8], Theorem 1.90],

$$
\left(x^{\lambda}\right)^{\Delta}=\lambda x^{\Delta} \int_{0}^{1}\left[x+\mu h x^{\Delta}\right]^{\lambda-1} d h>0 .
$$

Using (5.9) in (5.8) we have

$$
w^{\Delta}(t) \leq-\eta(t) q(t)+\eta^{\Delta}(t) \frac{\left(x^{\Delta^{n-1}}(t)\right)^{\sigma}}{\left(x^{\sigma}(t)\right)^{\lambda}} \leq-\eta(t) q(t)+\eta^{\Delta}(t) \frac{x^{\Delta^{n-1}}(t)}{x^{\lambda}(t)}, \quad t \geq t_{1}
$$

and hence in view of (5.5), we find

$$
w^{\Delta}(t) \leq-\eta(t) q(t)+\frac{\eta^{\Delta}(t)}{h_{n-1}\left(t, t_{1}\right)} x^{1-\lambda}(t), \quad t>t_{1} .
$$

Let $\lambda>1$. Since there exist $c>0$ and $t_{2} \geq t_{1}$ such that $x(t) \geq c$ for all $t \geq t_{2}$, we have $x^{1-\lambda}(t) \leq c^{1-\lambda}:=c_{1}$ for all $t \geq t_{2}$. If $\lambda=1$, then $x^{1-\lambda}(t)=1$ for all $t \geq t_{1}$. If $\lambda<1$, then there exist $b>0$ and $t_{3} \geq t_{1}$ such that $x^{\Delta^{n-1}}(t) \leq b$ for all $t \geq t_{3}$, and hence $x^{1-\lambda}(t) \leq c_{2} h_{n-1}^{1-\lambda}\left(t, t_{1}\right)$ for all $t \geq t_{3}$, where $c_{2}:=b^{1-\lambda}$. Combining all these we see that

$$
x^{1-\lambda}(t) \leq A\left(t, t_{1}\right), \quad t \geq t_{4}
$$

for some $t_{4} \geq \max \left\{t_{2}, t_{3}\right\}$. From (5.10) and (5.11),

$$
w^{\Delta}(t) \leq-\eta(t) q(t)+\eta^{\Delta}(t) \frac{A\left(t, t_{1}\right)}{h_{n-1}\left(t, t_{1}\right)}, \quad t \geq t_{4} .
$$

Integrating this inequality from $t_{4}$ to $t$, we find

$$
\int_{t_{4}}^{t}\left[\eta(s) q(s)-\eta^{\Delta}(s) \frac{A\left(s, t_{1}\right)}{h_{n-1}\left(s, t_{1}\right)}\right] \Delta s \leq w\left(t_{4}\right) .
$$

Taking limit superior as $t \rightarrow \infty$, we obtain a contradiction to condition (5.6). This completes the proof. $\square$

In the following result, we employ the lemma below, see [10].

Lemma 5.1. If $X$ and $Y$ are nonnegative and $\alpha>1$, then

$$
X^{\alpha}-\alpha X Y^{\alpha-1}+(\alpha-1) Y^{\alpha} \geq 0
$$

where equality holds if and only if $X=Y$. 
Theorem 5.2. Let condition (5.2) hold. If there exists a positive, nondecreasing, differentiable function $\eta \in C_{r d}\left(\mathbb{T}, \mathbb{R}^{+}\right)$such that for any $t_{1} \geq t_{0}$,

$$
\limsup _{t \rightarrow \infty} \int_{t_{1}}^{t}\left[\eta(s) q(s)-\frac{\left(\eta^{\Delta}(s)\right)^{\lambda+1}}{(\lambda+1)^{\lambda+1}\left(h_{n-2}\left(s, t_{0}\right) \eta(s) B\left(s, t_{0}\right)\right)^{\lambda}}\right] \Delta s=\infty,
$$

where

$$
B\left(t, t_{0}\right)=\left\{\begin{array}{lr}
c_{1}, c_{1} \text { is any positive constant, } & \text { when } \lambda>1 \\
1, & \text { when } \lambda=1 \\
c_{2}\left(h_{n-1}^{\sigma}\left(t, t_{0}\right)\right)^{\lambda+1}, c_{2} \text { is any positive constant, when } \lambda<1,
\end{array}\right.
$$

then Equation (5.1) is oscillatory.

Proof. Let $x(t)$ be a nonoscillatory solution of Equation (5.1), say, $x(t)>0$ for $t \geq t_{0}$. Let $w$ be as in (5.7). Then (5.8) and (5.9) hold. We also have

$$
w^{\Delta} \leq-\eta q+\frac{\eta^{\Delta}}{\eta} w^{\sigma}-\lambda \eta\left(\frac{x^{\Delta}}{x}\right)(w / \eta)^{\sigma} .
$$

Using the fact that $\ell=n-1$ and

$$
\frac{x^{\Delta^{n-1}}}{x} \geq\left(\left(\frac{w}{\eta}\right)^{\sigma}\right)^{1 / \lambda}\left(x^{\sigma}\right)^{\lambda-1}
$$

in (5.15), we obtain

$$
w^{\Delta} \leq-\eta q+\frac{\eta^{\Delta}}{\eta} w^{\sigma}-\lambda \eta h_{n-2}\left(\left(\frac{w}{\eta}\right)^{\sigma}\right)^{1+1 / \lambda}\left(x^{\sigma}\right)^{\lambda-1} .
$$

If $\lambda>1$, then from $x^{\sigma}(t) \geq x^{\sigma}\left(t_{1}\right)$ for $t \geq t_{1}$, we have $\left(x^{\sigma}(t)\right)^{\lambda-1} \geq c_{1}=\left(x^{\sigma}\left(t_{1}\right)\right)^{\lambda-1}$. In case $\lambda=1,\left(x^{\sigma}(t)\right)^{\lambda-1}=1$ for all $t \geq t_{1}$. Finally, let $\lambda<1$. We see that there exist $t_{2} \geq t_{1}$ and $b>0$ such that $x^{\Delta^{n-1}}(t) \leq b$ for all $t \geq t_{2}$. It follows that $x(t) \leq b h_{n-1}\left(t, t_{1}\right)$ for all $t$ $\geq t_{2}$, and hence $\left(x^{\sigma}(t)\right)^{\lambda-1} \geq b^{\lambda-1}\left(h_{n-1}^{\sigma}\left(t, t_{1}\right)\right)^{\lambda-1}$ for all $t \geq t_{2}$, where $c_{2}=b^{\lambda-1}$. Putting all these together, we have

$$
\left(x^{\sigma}(t)\right)^{\lambda-1} \geq B\left(t, t_{1}\right), \quad t \geq t_{2} .
$$

In view of (5.17) and (5.16), we find

$$
w^{\Delta}(t) \leq-\eta(t) q(t)+\frac{\eta^{\Delta}(t)}{\eta(t)} w^{\sigma}(t)-\lambda \eta(t) h_{n-2}\left(t, t_{1}\right) B\left(t, t_{1}\right)\left(\left(\frac{w(t)}{\eta(t)}\right)^{\sigma}\right)^{1+1 / \lambda}, \quad t \geq t_{2} .
$$

Now, setting

$$
X=\left(\lambda \eta h_{n-2} B\right)^{\lambda /(\lambda+1)}\left(\frac{w}{\eta}\right)^{\sigma} \text { and } Y=\left(\frac{\lambda}{\lambda+1}\right)^{\lambda}\left(\eta^{\Delta}\right)^{\lambda}\left(\left(\frac{1}{\lambda h_{n-2} B}\right)^{\lambda /(\lambda+1)}\right)^{\lambda}
$$

and $\alpha=(\lambda+1) / \lambda>1$ in Lemma 5.1, we have

$$
\lambda \eta h_{n-2} B\left(\left(\frac{w}{\eta}\right)^{\sigma}\right)^{1+1 / \lambda}-\eta^{\Delta}\left(\frac{w}{\eta}\right)^{\sigma}+\frac{\left(\eta^{\Delta}\right)^{\lambda+1}}{(\lambda+1)^{\lambda+1}\left(\eta h_{n-1} B\right)^{\lambda}} \geq 0 .
$$


Therefore, from (5.18)

$$
w^{\Delta} \leq-\eta q+\frac{1}{(\lambda+1)^{\lambda+1}}-\frac{\left(\eta^{\Delta}\right)^{\lambda+1}}{\left(\eta h_{n-1} B\right)^{\lambda}}, \quad t \geq t_{2} .
$$

Integrating this inequality from $t_{2}$ to $t$ results in

$$
\int_{t_{2}}^{t}\left[\eta(s) q(s)-\frac{1}{(\lambda+1)^{\lambda+1}} \frac{\left(\eta^{\Delta}(s)\right)^{\lambda+1}}{\left(\eta(s) h_{n-2}\left(s, t_{1}\right) B\left(s, t_{1}\right)\right)^{\lambda}}\right] \Delta s \leq w\left(t_{2}\right),
$$

which contradicts (5.15). This completes the proof. $\square$

Finally, we present the following result.

Theorem 5.3. Let condition (5.2) hold. If there exists a positive, nondecreasing differentiable function $\eta$ such that for any $t_{1} \geq t_{0}$,

$$
\limsup _{t \rightarrow \infty} \int_{t_{1}}^{t}\left[\eta(s) q(s)-\frac{\left(\eta^{\Delta}(s)\right)^{\lambda}}{4 \lambda \eta(s) B\left(s, t_{0}\right) h_{n-2}\left(s, t_{0}\right)\left(h_{n-1}^{\sigma}\left(s, t_{0}\right)\right)^{\lambda}}\right] \Delta s=\infty,
$$

where $B\left(t, t_{0}\right)$ is as in (5.14), then Equation (5.1) is oscillatory.

Proof. Let $x(t)$ be a nonoscillatory solution of Equation (5.1), say, $x(t)>0$ for $t \geq t_{0}$. Proceeding as in the proof of Theorem 5.2, we obtain

$$
\begin{aligned}
w^{\Delta} & \leq-\eta q+\eta^{\Delta}\left(\frac{w}{\eta}\right)^{\sigma}-\lambda \eta h_{n-2} B\left(\left(\frac{w}{\eta}\right)^{\sigma}\right)^{1+1 / \lambda} \\
& =-\eta q+\eta^{\Delta}\left(\frac{w}{\eta}\right)^{\sigma}-\lambda \eta h_{n-2} B \frac{\left(w^{\sigma}\right)^{1 / \lambda-1}}{\left(\eta^{\sigma}\right)^{1 / \lambda+1}}\left(w^{\sigma}\right)^{2},
\end{aligned}
$$

where $B=B\left(t, t_{1}\right)$ and $h_{n-2}=h_{n-2}\left(t, t_{1}\right)$. Since

$$
w^{1 / \lambda-1}(t)=\lambda^{1 / \lambda-1}\left(\frac{x^{\Delta^{n-1}}(t)}{x(t)}\right)^{1-\lambda} \geq \eta^{1 / \lambda-1}(t) h_{n-1}^{\lambda-1}\left(t, t_{1}\right),
$$

it follows that

$$
\begin{aligned}
w^{\Delta} \leq & -\eta q+\eta^{\Delta}\left(\frac{w}{\eta}\right)^{\sigma}-\lambda \eta B h_{n-1}\left(h_{n-1}^{\sigma}\right)^{\lambda-1}\left(\frac{w^{\sigma}}{\eta^{\sigma}}\right)^{2} \\
= & -\eta q-\left[\left(\lambda \eta B h_{n-2}\left(h_{n-1}^{\sigma}\right)^{\lambda-1}\right)^{1 / 2}\left(\frac{w}{\eta}\right)^{\sigma}-\frac{\eta^{\Delta}}{2\left(\lambda \eta B h_{n-2}\left(h_{n-1}^{\sigma}\right)^{\lambda-1}\right)^{1 / 2}}\right]^{2} \\
& +\frac{\left(\eta^{\Delta}\right)^{2}}{4 \lambda \eta B h_{n-2}\left(h_{n-1}^{\sigma}\right)^{\lambda-1}} \\
\leq & -\eta q+\frac{\left(\eta^{\Delta}\right)^{2}}{4 \lambda \eta B h_{n-2}\left(h_{n-1}^{\sigma}\right)^{\lambda-1}}, \quad t \geq t_{2} .
\end{aligned}
$$


Integrating this inequality from $t_{2}$ to $t$, we have

$$
\int_{t_{2}}^{t}\left[\eta(s) q(s)-\frac{\left(\eta^{\Delta}(s)\right)^{2}}{4 \lambda \eta(s) B\left(s, t_{1}\right) h_{n-2}\left(s, t_{1}\right)\left(h_{n-1}^{\sigma}\left(s, t_{1}\right)\right)^{\lambda-1}}\right] \Delta s \leq w\left(t_{2}\right),
$$

which contradicts (5.19). This completes the proof. $\square$

Remark 3. We note that the oscillation criteria given in this article are new for the corresponding difference equations and some of these results are new for the corresponding differential and/or delay differential equations. The results can be extended easily to equations of the form

$$
x^{\Delta^{n}}(t)+f(t, x(\xi(t))=0,
$$

when $f: \mathbb{T} \times \mathbb{R} \rightarrow \mathbb{R}$ is continuous and $f$ is strongly superlinear or $f$ is strongly sublinear, see [4].

As examples, we have reformulated some of the obtained results for the time-scales $\mathbb{T}=\mathbb{R}$ (i.e., the continuous case) and $\mathbb{T}=\mathbb{Z}$ (i.e., the discrete case). One may obtain more results by employing other types of time scales such as $\mathbb{T}=h \mathbb{Z}$ with $h>0$, $\mathbb{T}=q^{\mathbb{N}_{0}}$ with $q>1$, and $\mathbb{T}=\mathbb{N}_{0}^{2}$, see [8]. The details are left to the reader.

\section{Author details}

${ }^{1}$ Department of Engineering Mathematics, Faculty of Engineering, Cairo University, Oman, Giza 12221, Egypt

${ }^{2}$ Department of Mathematics, Texas A\&M University-Kingsville, Kingsville, TX 78363, USA ${ }^{3}$ Department of Mathematics, Middle East Technical University, 06800 Ankara, Turkey

\section{Authors' contributions}

The authors declare that the study was realized in collaboration with the same responsibility. All authors read and approved the final manuscript.

\section{Competing interests}

The authors declare that they have no competing interests.

Received: 7 February 2012 Accepted: 23 May 2012 Published: 23 May 2012

\section{References}

1. Agarwal, RP, Anderson, DR, Zafer, A: Interval oscillation criteria for second-order forced delay dynamic equations with mixed nonlinearities. Comput Math Appl. 59, 977-993 (2010). doi:10.1016/j.camwa.2009.09.010

2. Anderson, DR, Zafer, A: Interval criteria for second-order super-half-linear functional dynamic equations with delay and advance arguments. J Diff Equ Appl. 16, 917-930 (2010). doi:10.1080/10236190802582134

3. Erbe, L, Peterson, A, Saker, S: Kamenev type oscillation criteria for second-order linear delay dynamic equations. Dyn. Sys Appl. 15, 65-78 (2006)

4. Grace, SR, Agarwal, RP, Bohner, M, O'Regan, D: Oscillation of second-order strongly superlinear and strongly sublinear dynamic equations. Commun Nonlinear Sci Numer Simul. 14, 3463-3471 (2009). doi:10.1016/j.cnsns.2009.01.003

5. Grace, SR, Bohner, M, Agarwal, RP: On the oscillation of second-order half-linear dynamic equations. J Diff Equ Appl. 15 451-460 (2009). doi:10.1080/10236190802125371

6. Grace, SR, Agarwal, RP, Kaymakcalan, B, Wichuta, W: On the oscillation of certain second-order nonlinear dynamic equations. Math Comput Model. 50, 273-286 (2009). doi:10.1016/j.mcm.2008.12.007

7. Unal, M, Zafer, A: Oscillation of second-order mixed-nonlinear delay dynamic equations. Adv Diff Equ 2010, 21 (2010). (Art. ID 389109)

8. Bohner, M, Peterson, A: Dynamic Equations on Time-Scales: An Introduction with Applications. Birkhäuser, Boston (2001)

9. Hilger, S: Analysis on measure chains-a unified approach to continuous and discrete calculus. Results Math. 18, 18-56 (1990)

10. Hardy, GH, Littlewood, JE, Polya, G: Inequalities. Cambridge University Press, Cambridge, 2 (1998)

doi:10.1186/1687-1847-2012-67

Cite this article as: Grace et al:: Oscillation of higher order nonlinear dynamic equations on time scales. Advances in Difference Equations 2012 2012:67. 